\title{
microRNA-27a functions as a tumor suppressor in esophageal squamous cell carcinoma by targeting KRAS
}

\author{
LINAN ZHU $^{1}$, ZHIJU WANG ${ }^{2}$, QINGXIA FAN ${ }^{1}$, RUILIN WANG ${ }^{1}$ and YAN SUN ${ }^{1}$ \\ ${ }^{1}$ Department of Oncology, The First Affiliated Hospital of Zhengzhou University, Zhengzhou, Henan; \\ ${ }^{2}$ Department of Basic Medicine, Zhengzhou University, Zhengzhou, Henan, P.R. China
}

Received September 3, 2013; Accepted October 4, 2013

DOI: 10.3892/or.2013.2807

\begin{abstract}
RNAs (miRNAs) have been suggested to play a vital role in regulating tumor progression and invasion. However, the expression of miR-27a in esophageal squamous cell carcinoma (ESCC) and its effect on the tumorigenesis of ESCC are unclear. In the present study, we found that miR-27a was downregulated in esophageal carcinoma cell lines and ESCC specimens with lymph node metastasis. Furthermore, we demonstrated that miR-27a binds to the 3'-untranslated region (UTR) of KRAS and inhibits the expression of the KRAS protein. miR-27a levels were inversely correlated with levels of KRAS mRNA and protein in ESCC specimens. Both in vitro and in vivo assays revealed that miR-27a attenuated ESCC proliferation, invasion and tumor growth in nude mice. miR-27a exerts its tumor suppressor function through inhibition of the KRAS-related ERK pathways. Our findings suggest, for the first time, that miR-27a suppresses tumorigenesis of ESCC by targeting KRAS.
\end{abstract}

\section{Introduction}

microRNAs (miRNAs) are an endogenous conserved class of non-coding 20-22 nt small RNAs that regulate gene expression at the post-transcriptional level by mostly binding to 3'-UTR of target mRNAs, leading to mRNA degradation or translation inhibition. Recent reports demonstrate a role for miRNA expression in disease progression and outcome. To date, several miRNA expression profiles in esophageal squamous cell carcinoma (ESCC) have been reported $(1,2)$, and several specific miRNAs have been proven to be involved in ESCC tumorigenesis, including miR-21 (3) and miR-129-2 (4).

The role of miR-27a in tumorigenesis differs in various cells and tissues. It is regarded as an oncogene in several types of tumors. Liu et al (5) reported that suppression of miR-27a

Correspondence to: Professor Yan Sun, Department of Oncology, The First Affiliated Hospital of Zhengzhou University, Kexue Road 100, Zhengzhou, Henan 450001, P.R. China

E-mail: sunyzzu@163.com

Key words: miR-27a, esophageal squamous cell carcinoma, tumor suppressor, KRAS inhibits gastric cancer cell growth by targeting prohibitin; Zhang et al (6) confirmed that the overexpression of miR-27 promotes the metastasis of the human gastric cancer cell line AGS, whereas its depletion decreases cell metastasis. However, miR-27a was found to be downregulated in several other tumor types (reviewed in ref. 7), including acute promyelocytic leukemia (8), colorectal cancer $(9,10)$, malignant melanoma (11), oral squamous cell carcinoma (12) and prostate cancer (13), indicating it may be a possible tumor suppressor. Most recently, miR-27a was found to target epidermal growth factor receptor (EGFR) $(14,15)$, which is activated and promotes tumorigenesis in numerous types of tumors.

To date, there are no report on the role of miR-27a in the tumorigenesis of ESCC. Although Zhang et al (16) showed that the downregulation of miR-27a reversed the multidrug resistance of ESCCs, they did not investigate the expression level of miR-27a in ESCC and its possible role in tumorigenesis.

In the present study, we evaluated the expression of miR-27a in ESCC specimens and cell lines and studied the role of miR-27a in cell growth and migration of esophageal carcinoma cell line TE-1. We also investigated the mechanisms of miR-27a modulation during TE-1 cell growth.

\section{Materials and methods}

Cell lines. Primary cultures of normal esophageal epithelial cells (NEECs) were established in our laboratory. NEECs were established from fresh biopsies of adjacent noncancerous esophageal tissue, according to a previous report (17). The NEECs and ESCCs were grown at $37^{\circ} \mathrm{C}$ in $5 \% \mathrm{CO}_{2}$ with keratinocyte serum-free medium, with $40 \mu \mathrm{g} / \mathrm{ml}$ bovine pituitary extract, $1.0 \mathrm{ng} / \mathrm{ml}$ epidermal growth factor, $100 \mathrm{U} / \mathrm{ml}$ penicillin and $100 \mu \mathrm{g} / \mathrm{ml}$ streptomycin.

The esophageal cancer cell lines, including NECC, TE1, TE13, Eca109, EC9706, KYSE140 and KYSE30, were obtained from the Cell Bank of the Type Culture Collection of the Chinese Academy of Sciences (Shanghai, China). All cell lines were grown in RPMI-1640 medium (Invitrogen, Carlsbad, CA, USA) supplemented with $10 \%$ fetal bovine serum, $100 \mu \mathrm{g} / \mu \mathrm{l}$ streptomycin and $100 \mu \mathrm{g} / \mu \mathrm{l}$ penicillin in a humidified incubator containing $5 \% \mathrm{CO}_{2}$ at $37^{\circ} \mathrm{C}$.

Patient information and tissue specimens. The present study was conducted on a total of 25 samples from ESCC patients 
with nodal involvement, which were histopathologically and clinically diagnosed at the First Affiliated Hospital of Zhengzhou University in 2012. For the use of these clinical materials for research purposes, prior patient consent and approval from the Institutional Research Ethics Committee were obtained.

Quantitative RT-PCR analysis ( $q R T-P C R)$. Total RNAs were extracted from cells with TRIzol reagent (Invitrogen). For the detection of KRAS mRNA, cDNA was synthesized from $1 \mathrm{mg}$ of total RNA by means of a reverse reaction kit according to the manufacturer's instructions (Promega, Madison, WI, USA). Human GAPDH was amplified in parallel as an internal control. For miR-27a, reverse transcription and qRT-PCR reactions were performed by means of a qSYBR-Green-containing PCR kit (Shanghai Genepharma Co., Ltd., Shanghai, China), and U6 snRNA was used as an endogenous control for miRNA detection. Expression of each gene was quantified by measuring cycle threshold $(\mathrm{Ct})$ values and normalized using the $\Delta \mathrm{Ct}$ method $\left[2^{\mathrm{Ct}(\text { reference })}-\mathrm{Ct}(\right.$ target $\left.)\right]$ relative to U6 snRNA or GAPDH.

Constructs. luc-UTR vectors were constructed by cloning the predicted miR-27a target region or its mutant control into the NheI and SalI sites of the pmirGLO luciferase vector (Promega) using the PCR generated fragments. The oligonucleotide pairs contained the Kpn1 internal site for clone confirmation: sense-wt: 5'-CTAGCTAGGTACCTTGAACT AGCAATGCCTGTGAAAG-3' and antisense-wt: 5'-TCG ACTTTCACAGGCATTGCTAGTTCAAGGTACCTAG-3'; sense-mut: 5'-CTAGCTAGGTACCTTATTATAGCAATG CACACAGAAG-3' and antisense-mut: 5'-TCGACTTCTGT GTGCATTGCTATAATAAGGTACCTAG-3'. Bold indicates NheI and SalI sites; underlining indicates the Kpn1 site; italics indicates the mutated sites.

Synthesized RNA duplexes of scramble miRNA, miR-27a, and their inhibitors anti-scramble and anti-miR-27a were obtained from Baoxin Bio-Technology Co., Ltd. (Zhengzhou, China). To construct a vector expressing miR-27a, the precursor sequence of miR-27a (MI0000085) was synthesized, annealed and then inserted into the BamHI-HindIII fragment of the pGCsi/U6 vector (GeneChem, Shanghai, China). A construct including the non-specific miRNA was used as a negative control. The miR-27a knockdown lentivirus was purchased from Baoxin Bio-Technology.

The KRAS-expressing vector was constructed by cloning full-length KRAS cDNA into the eukaryotic expression vector pcDNA3.1(+) (Invitrogen). The empty pcDNA3.1(+) vector was used as a negative control.

Cell transfection and infection. TE-1 cells were infected with the miR-27a lentivirus or the control lentivirus expressing a scrambled miRNA. All cells were selected with $500 \mathrm{mg} / \mathrm{l}$ G418 to generate two stable monoclonal cell lines (a stable cell line expressing miR-27a, TE-1-miR-27a and a control stable cell line, TE-1-scramble).

To establish stable miR-27a knockdown cell lines, TE-1 cells were transduced with the miR-27a knockdown lentivirus or the control lentivirus and selected with $5 \mathrm{mg} / \mathrm{l}$ puromycin.
For miRNA and pcDNA3.1-KRAS combination experiments, TE-1-miR-27a and TE-1-scramble cells were transfected with pcDNA3.1-KRAS or empty vector using Lipofectamine 2000 (Invitrogen).

Luciferase assay. TE-1-miR-27a cells were transfected with pmirGLO-KRAS-wt, pmirGLO-KRAS-mut or pmirGLO-ctrl using Lipofectamine 2000 (Invitrogen). Luciferase activity was measured $24 \mathrm{~h}$ after transfection using the Dual-Glo luciferase assay system (Promega). The Renilla luciferase activity served as internal control.

MTT assay. Cells were seeded onto 96-well plates at a density of $5 \times 10^{4}$ cells/well in $100 \mu \mathrm{l}$ medium. All cells were maintained in a humidified $37^{\circ} \mathrm{C}$ incubator with $5 \% \mathrm{CO}_{2} .20 \mu \mathrm{l}$ 3-(4,5-Dimethylthiazol-2-yl)-2,5-diphenyltetrazolium bromide (MTT) solution ( $5 \mathrm{~g} / \mathrm{l}$ in phosphate-buffered saline) was added to each well of the microplate, and the absorbance at $570 \mathrm{~nm}$ was measured by a microplate reader. After a 4-h incubation, the number of viable cells was measured a point, was set 5 re-wells.

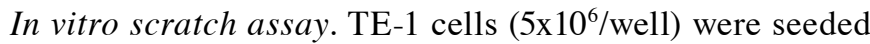
to $90 \%$ confluence in a 6 -well plate for overnight culture. The following day a scratch was made through the center of each well using a 200- $\mu$ l pipette tip, creating an open 'scratch' or 'wound' that was clear of cells. The dislodged cells were removed by three washes with complete culture media, and cells were incubated under standard conditions. Migration into the open area was documented at $72 \mathrm{~h}$ postscratching.

Western blot analysis. Western blot analyses were performed as previously described (18). Antibodies against KRAS (Sigma-Aldrich), ERK (Santa Cruz Biotechnology), phosphorylated ERK (Santa Cruz Biotechnology), and c-Fos (Santa Cruz Biotechnology) were obtained from Cell Signaling Technology. The anti- $\beta$-actin antibody was from Santa Cruz Biotechnology.

In vivo tumorigenesis. Five-week-old male nude athymic $\mathrm{BALB} / \mathrm{c} \mathrm{nu} / \mathrm{nu}$ mice were used for examining tumorigenicity. To evaluate the role of miR-27a in tumor formation, TE-1miR-27a cells, TE-1-scramble control, TE-1-anti-miR-27a cells, or TE-1-anti-scramble control were propagated and inoculated subcutaneously into the dorsal flanks of nude mice $\left(2 \times 10^{6}\right.$ cells in a $0.2-\mathrm{ml}$ volume $)$. Tumor size was measured every 5 days. After 30 days, the mice were sacrificed, necropsies were performed and the tumors were weighed. Tumor volumes were determined according to the following formula: $\mathrm{A} \times \mathrm{B}^{2} / 2$, where $\mathrm{A}$ is the largest diameter and $\mathrm{B}$ is the diameter perpendicular to $\mathrm{A}$. The experiments were performed using five mice per group, and all animal procedures were performed in accordance with institutional guidelines.

Statistical analysis. Statistical evaluation of data was performed using SPSS 13 analysis software (SPSS, Chicago, IL, USA). Independent-samples t-test, or paired-samples t-test were used to evaluate statistical significance. Spearman's correlation tests were used to evaluate the pair-wise expression 

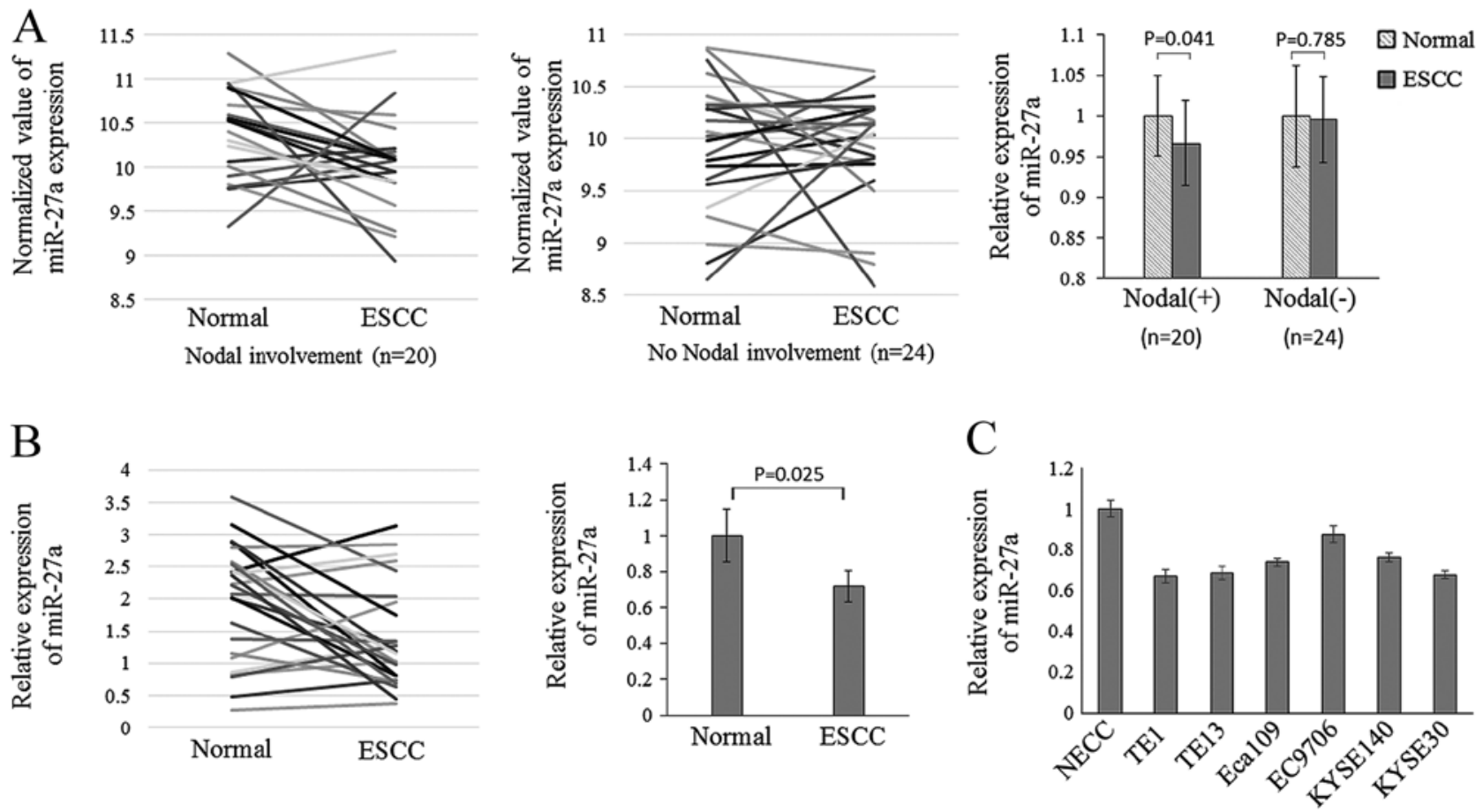

Figure 1. miR-27a is downregulated in ESCC tissues and cell lines. (A) Analysis of miR-27a expression in ESCC tissues. The published miRNA array data of 44 ESCC specimens was downloaded from GSE13937. The normalized value of miR-27a is shown and was analyzed. In the histogram, the expression value of paired adjacent normal tissue was set to 1. (B) qRT-PCR analysis of the expression levels of miR-27a in 25 ESCC specimens with nodal involvment and their paired adjacent normal tissues. In the line chart, the relative expression levels of miR-27a were calculated using the $\Delta \mathrm{CT}$ method [value $=2^{\mathrm{CT}(\mathrm{U} 6) \text { - } \mathrm{CT}(\mathrm{miR}-27 \mathrm{a})}$ ] $\mathrm{In}$ the histogram, the miR-27a expression value of normal tissue was set to 1 , and the relative expression levels of miR-27a were determined by using the $2^{-\Delta \Delta C T}$ method. (C) qRT-PCR analysis of the expression levels of miR-27a in multiple esophageal carcinoma cell lines. The expression values of the NEECs were set to 1 . The relative expression levels of miR-27a were determined by using the $2^{-\Delta \Delta C T}$ method. All data are shown as means \pm SEM. NEECs, normal esophageal epithelial cells; ESCC, esophageal squamous cell carcinoma.

correlation between miR-27a and KRAS. Data are shown as means \pm SEM. The significant level was set at $\mathrm{P}<0.05$.

\section{Results}

miR-27a is downregulated in ESCC cell lines and clinical specimens. Published datasets archived in the publicly available Gene Expression Omnibus (GEO) repository were used for re-analysis using GEO2R (NCBI online gene expression tool) (19). Datasets published in GEO reference series GSE13937 (20) were used for microRNA expression analysis of hsamiR-27a in ESCC compared with adjacent normal esophageal tissues. These datasets used OSU-CCC Human and Mouse MicroRNA Microarray version 3.0 array platform. Normalized signal intensity value was analyzed using the paired-samples t-test analysis. The expression of hsa-miR-27a was decreased in tumors when compared with that in adjacent normal tissues from ESCC patients with nodal involvement $(n=20 ; P=0.041)$. However, in ESCC patients without nodal involvement, this difference was not significant $(n=24 ; P=0.785)$ (Fig. 1A).

We used quantitative real-time PCR (qRT-PCR) to measure mature miR-27a expression levels in ESCC tissues of patients with nodal involvement and cell lines. miR-27a was significantly downregulated in ESCC tissues when compared with the paired adjacent normal tissues (paired-samples t-test, $\mathrm{n}=25 ; \mathrm{P}=0.025$ ) (Fig. 1B). In six esophageal carcinoma cell lines, the expression of miR-27a was decreased, particularly in TE-1 cells (independent-samples t-test; $\mathrm{P}=0.003$ ) (Fig. 1C).
miR-27a directly targets and inhibits KRAS. To determine the role of miR-27a in ESCCs, we performed a bioinformatic search (TargetScan and PicTar) to identify putative mRNA 3'-UTR targets for the mature miR-27a. We found that miR-27a had a seed region that matched the 3'-UTR of human KRAS (nucleotides 343-349; NM_033360) (Fig. 2A). To verify that KRAS is a direct target of miR-27a, the KRAS 3'-UTR containing the miR-27a binding site was cloned into the pmirGLO control vector downstream of the luciferase ORF. This reporter construct was used to transfect 293 cells. Co-transfection of miR-27a with the wt KRAS 3'-UTR construct in 293 cells resulted in a significant inhibition of luciferase activity compared with the negative control (Fig. 2B). Mutagenesis of the miR-27a binding site within the KRAS 3'-UTR abolished the ability of miR-27a to regulate the luciferase expression (Fig. 3B). In addition, overexpression of miR-27a in TE-1 cells strongly reduced the endogenous protein and mRNA levels of KRAS when compared with these levels in the control (Fig. 2C and D). In clinical ESCC specimens, there was an inverse correlation between the mRNA/protein levels of KRAS and the level of miR-27a expression (Fig. 2E). These data suggest that miR-27a acts as a tumor suppressor by targeting KRAS in ESCC.

miR-27a inhibits the ERK pathway. KRAS activation can trigger several important signaling pathways, such as the Ras/Raf/MEK/ERK pathways, most of which regulate cell proliferation, survival and invasion. Therefore, we investi- 
A

Position 343-349 of KRAS 3' UTR 5 ' $\ldots$ UUGAaCUAGCAAUGCCUGUGAAA. .

hsa-miR-27a

KRAS mutation

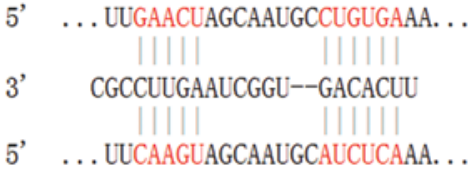
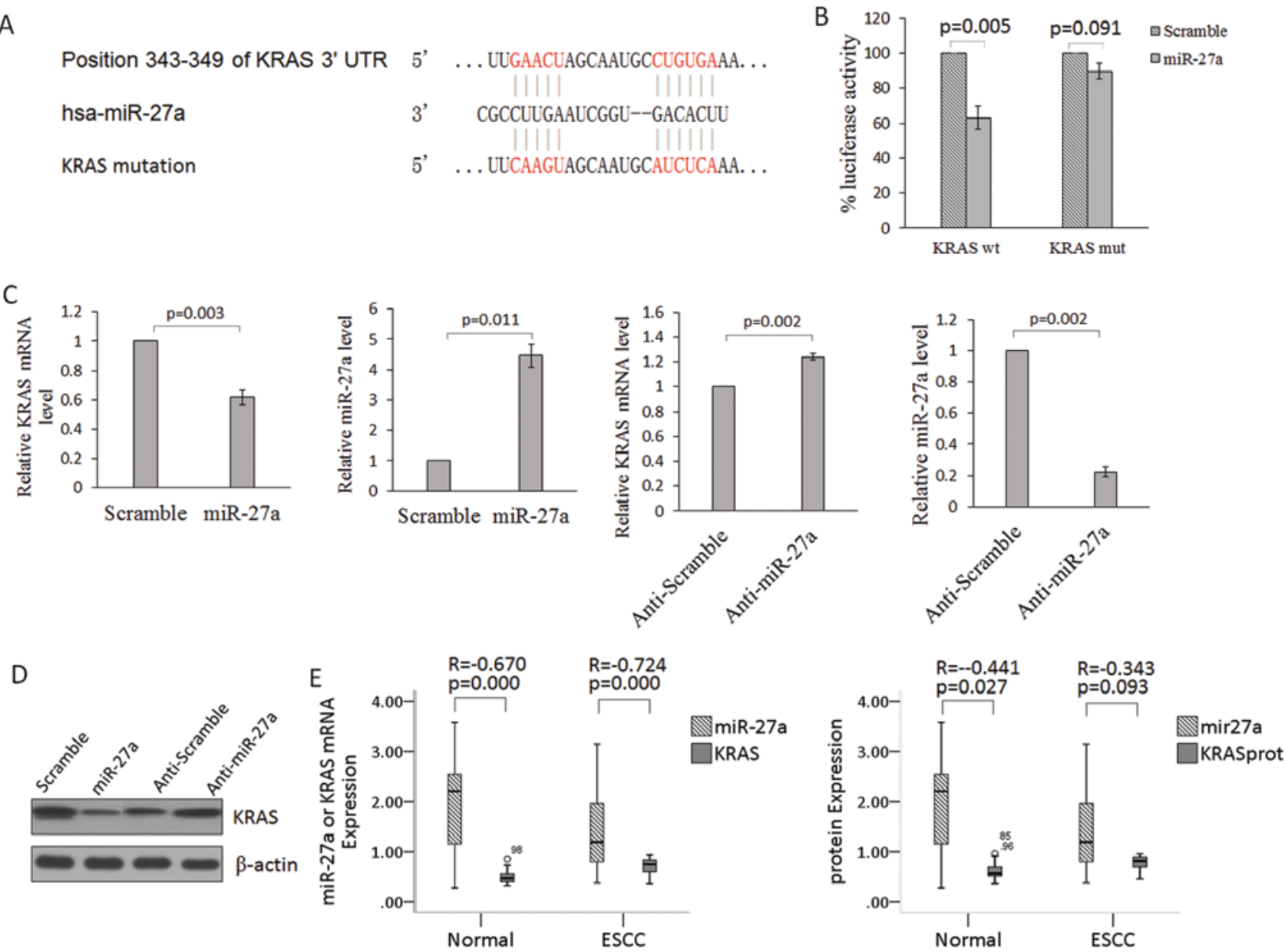

Figure 2. miR-27a targets KRAS in ESCCs. (A) Predicted KRAS 3'-UTR binding sites for miR-27a. The alignment of the seed regions of miR-27a with KRAS 3'-UTR are shown. The sites of target deletion mutagenesis are indicated in red.(B) KRAS 3'-UTR is a target of miR-27a. pmirGLO luciferase construct containing a wild-type (wt) or mutated (mut) KRAS 3'-UTR were co-transfected with miR-27a or scramble in 293 cells, and the luciferase assay was performed. Data were from 3 independent experiments and analyzed by simple-sample t-test. (C) Gain and loss of miR-27a function altered KRAS mRNA expression in TE-1 cells. TE-1 cells were transfected with miR-27a, scramble, anti-miR-27a (inhibitor) or anti-scramble for $72 \mathrm{~h}$. The mRNA levels of KRAS and miR-27a were assessed by qRTPCR. (D) Gain and loss of miR-27a function altered KRAS protein expression in TE-1 cells. TE-1 cells were transfected with miR-27a, scramble, anti-miR-27a (inhibitor) or anti-scramble for $72 \mathrm{~h}$. The protein level of KRAS was assessed by western blot analysis. (E) Pearson's correlation scatter plot of the levels of mature miR-27a, KRAS mRNA and KRAS protein in ESCCs. Twenty-five ESCC specimens (tumors and their adjacent normal tissues) were analyzed by qRT-PCR and western blot analysis. The expression levels of miR-27a and KRAS mRNA were calculated using the $\Delta \mathrm{CT}$ method [value $=2^{\mathrm{CT}(\text { (reference) }- \text { CT(target) }}$. The expression levels of KRAS protein were determined by relative gray level (KRAS/ $\beta$-actin). All data are shown as means \pm SEM.
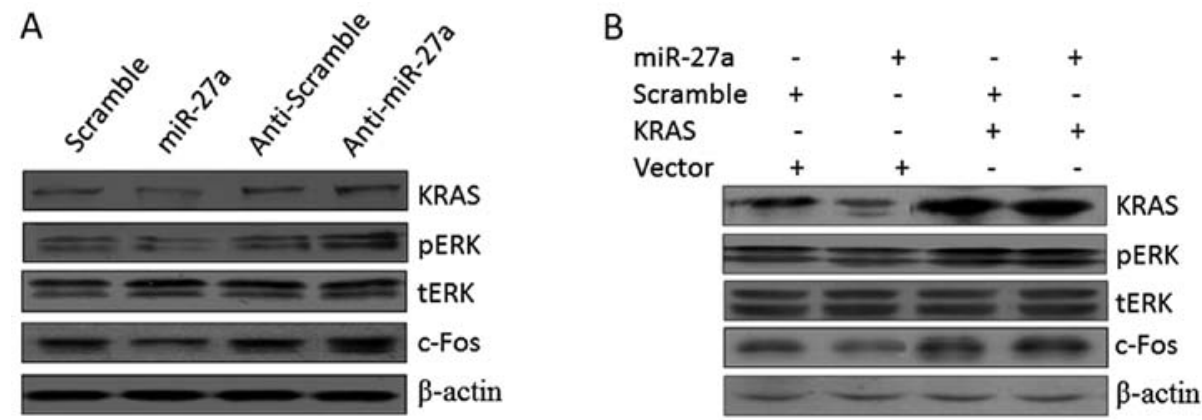

Figure 3. miR-27a inhibits ERK pathways by targeting KRAS. (A) Gain and loss of miR-27a function altered the activity of ERK pathways in TE-1 cells. (B) miR-27a transfection (or scramble as control) followed by KRAS (or mock vector as control) transfection $48 \mathrm{~h}$ later in TE-1 cells affected ERK signaling. The ERK pathway activity was measured by examining expression of phosphorylated ERK (pERK) and c-Fos.

gated the possibility that miR-27a regulates those pathways by targeting KRAS. Upregulation of miR-27a through transfection of miR-27a in TE-1 cells suppressed the levels of phosphorylated ERK and its downstream effector c-Fos. We also observed that knockdown of miR-27a, through transfection of anti-miR-27a, in TE-1 cells increased the 

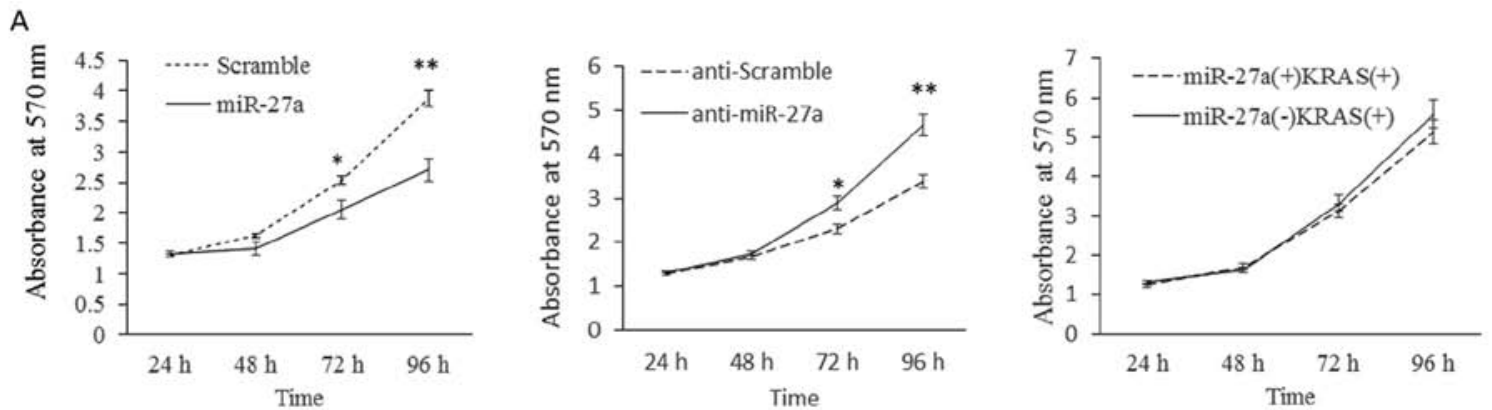

B

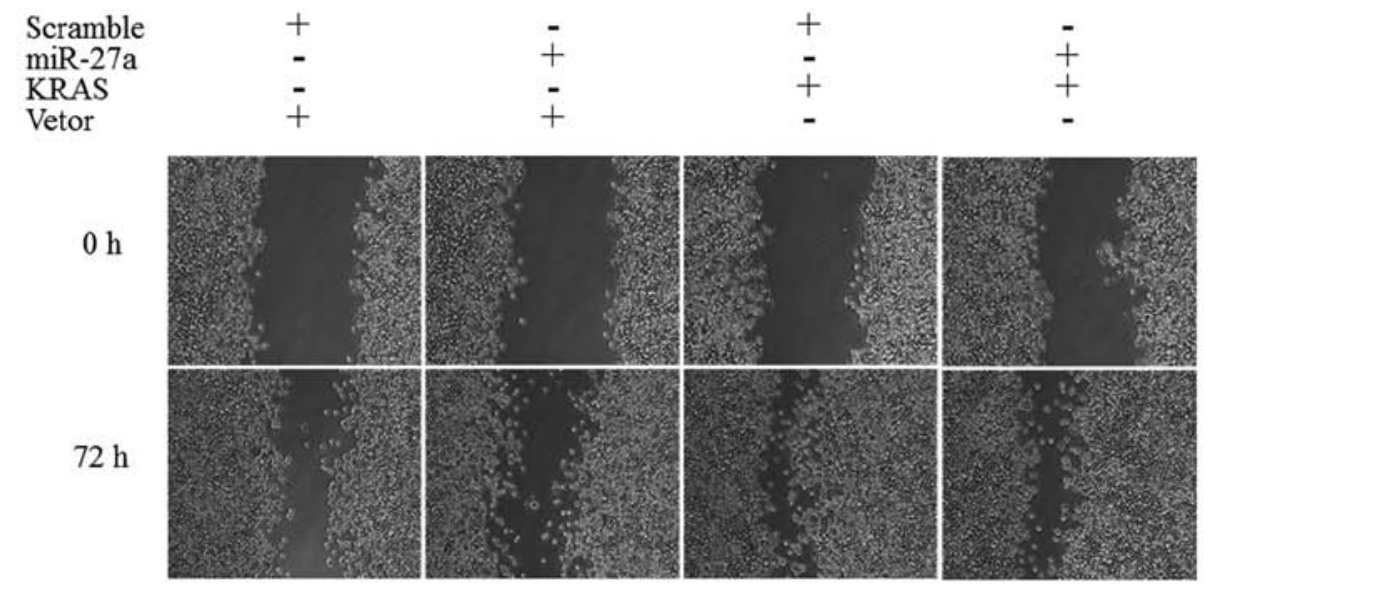

C
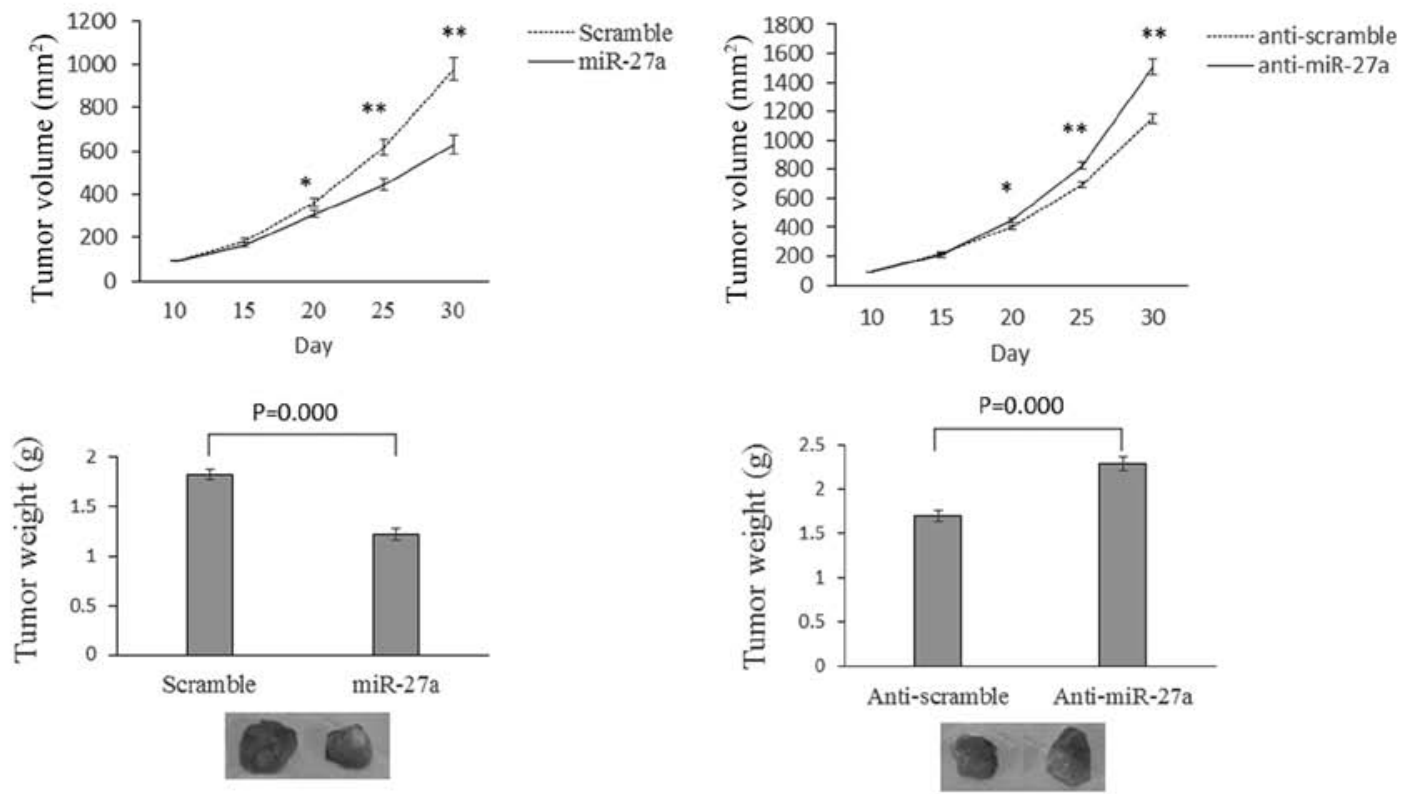

Figure 4. miR-27a inhibits cell proliferation, invasion and tumor growth. (A) Effect of miR-27a on cell proliferation as determined by MTT assay. Overexpression of miR-27a markedly attenuated cell proliferation, and knockdown of miR-27a significantly promoted cell growth in TE-1 cells. Forced expression of KRAS (without an endogenous 3'-UTR) partially rescued the cell growth inhibition by miR-27a. Data were from 3 independent experiments. (B) Effect of miR-27a on cell migration in a scratch assay. TE-1 cells were transfected with a lentivirus containing miR-29a precursor or control for 4 days and were seeded in 6-well plates. After cells had grown to confluence, a scratch was made in the monolayer. Images were recorded at $72 \mathrm{~h}$ at $\mathrm{x} 40 \mathrm{magnification}$. (C) miR-27a attenuated tumor growth in mouse xenograft models. The left panels show tumor formation upon subcutaneous injection of TE-1 cells that stably expressed miR-27a or scramble into nude mice. The right panels show tumor formation upon transplantation of TE-1 cells infected with the lentivirus expressing the miR-27a inhibitor or control sequence into nude mice. Tumor size was measured every 5 days. After 30 days, the mice were sacrificed, necropsies were performed and tumors were weighed. All data are shown as means \pm SEM. ${ }^{*} \mathrm{P}<0.05 ;{ }^{* * *} \mathrm{P}<0.01$.

levels of ERK (Fig. 3A). Results of the western blot analysis demonstrated that miR-27a is a negative regulator of the ERK pathway.

Subsequently, rescue experiments were performed by overexpressing the KRAS vector (without an endogenous 3'-UTR) in miR-27a-treated cells. TE-1 cells were first transfected with miR-27a and then with the KRAS-encoding vector $48 \mathrm{~h}$ later. The miR-27a-induced downregulation of KRAS was rescued upon the introduction of KRAS, and the phosphorylation level of ERK was altered in a similar manner (Fig. 3B). These 
observations suggest that miR-27a inhibits the ERK pathway by targeting KRAS.

miR-27a suppresses TE-1 cell proliferation and invasion by targeting KRAS. Overexpression of miR-27a markedly attenuated cell proliferation, and knockdown of miR-27a promoted cell proliferation in TE-1 cells (Fig. 4A). Forced expression of KRAS (without an endogenous 3'-UTR) rescued the cell growth inhibition of miR-27a partially, suggesting that miR-27a regulates cell growth through targeting KRAS (Fig. 4A). Scratch assays were used to measure cell migration or to observe the healing of scratches in cancer cell monolayers. Overexpression of miR-27a significantly inhibited the ability of TE-1 cells to heal scratch assays. However, knockdown of miR-27a significantly promoted this behavior (Fig. 4B). In nude mouse xenograft models, TE-1 cells with miR-27a overexpression developed smaller tumors (both in tumor volume and weight) compared with TE-1 cells with normal miR-27a (scramble). Accordingly, miR-27a knockdown in TE-1 cells promoted tumor formation (both in tumor volume and weight) in the nude mouse xenograft experiments (Fig. 4C).

\section{Discussion}

We first analyzed the miR-27a expression data archived in GEO. GSE13937 recorded the miRNA array data to compare miRNA expression between ESCC and paired adjacent normal tissues. We found that miR-27a was significantly downregulated in ESCCs with nodal involvement. However, miR-27a was not downregulated in ESCCs without nodal involvement. The downregulation of miR-27a is present only in ESCC specimens with nodal involvement, indicating it may participate in the migratory capacity of ESCC. In order to confirm the expression change of miR-27a, we further studied the miR-27a expression in 25 ESCC specimens with nodal involvement using qRT-PCR, and demonstrated miR-27a is downregulated in ESCCs. In six esophageal carcinoma cell lines, the expression of miR-27a was also decreased, particularly in TE-1 cells.

We next explored the possible targets of miR-27a in ESCCs through different computational algorithms. In the present study, we proved that miR-27a targets KRAS directly. KRAS is a GTPase and an early player in many signal transduction pathways. Active GTP-bound KRAS associates with a wide variety of effectors, including Raf, PI3K, Ral-GDS, Rho GTPases and other molecules, to transmit downstream signals that control distinct cellular events, including cell proliferation, survival, differentiation and invasion $(21,22)$. KRAS promotes tumorigenesis and has been proven to be downregulated by the let-7 family (23) and miR-96 (24). ESCC patients (16\%) (5/30 cases) were found to harbor KRAS gene mutations (25). In the present study, we demonstrated that miR-27a may be a negative regulator of the ERK pathway, through targeting KRAS and decreased expression of phosphorylated ERK and its downstream effector c-Fos.

In order to investigate the role of miR-27a in ESCCs, gain and loss of function experiments were conducted. Forced expression of miR-27a significantly decreased the cell proliferation and migration of TE-1 cells, while miR-27a inhibition exerted an opposite effect. In the nude mouse assay, the result was consistent with that in vitro. We hypothesized that miR-27a is a tumor suppressor in ESCCs, although it is regarded as an oncogene in several other tumor types $(5,6)$. Our results support the opinion that the same miRNA can have antagonizing roles in two different cell types; i.e. in one cell type the miRNA promotes proliferation whereas in another cell type the same miRNA inhibits proliferation (7).

In conclusion, this is the first study to show that miR-27a inhibits cell proliferation and invasion in ESCC cells. It is also the first study to show that tumor promotor KRAS is negatively regulated by miR-27a at the post-transcriptional level via binding to 3'-UTR of KRAS mRNA in esophageal squamous cell carcinoma cells.

\section{Acknowledgements}

The present study was supported by the Zhengzhou University 211 Project-Phase II, The Basic and Clinical Research of Stem Cells.

\section{References}

1. Gu J, Wang Y and Wu X: MicroRNA in the pathogenesis and prognosis of esophageal cancer. Curr Pharm Des 19: 1292-1300, 2013.

2. Yang M, Liu R, Sheng J, et al: Differential expression profiles of microRNAs as potential biomarkers for the early diagnosis of esophageal squamous cell carcinoma. Oncol Rep 29: 169-176, 2013.

3. Liu F, Zheng S, Liu T, et al: MicroRNA-21 promotes the proliferation and inhibits apoptosis in Eca109 via activating ERK1/2/ MAPK pathway. Mol Cell Biochem 381: 115-125, 2013.

4. Kang M, Li Y, Liu W, et al: miR-129-2 suppresses proliferation and migration of esophageal carcinoma cells through downregulation of SOX4 expression. Int J Mol Med 32: 51-58, 2013.

5. Liu T, Tang H, Lang Y, Liu M and Li X: MicroRNA-27a functions as an oncogene in gastric adenocarcinoma by targeting prohibitin. Cancer Lett 273: 233-242, 2009.

6. Zhang Z, Liu S, Shi R and Zhao G: miR-27 promotes human gastric cancer cell metastasis by inducing epithelial-to-mesenchymal transition. Cancer Genet 204: 486-491, 2011.

7. Chhabra R, Dubey R and Saini N: Cooperative and individualistic functions of the microRNAs in the miR-23a 27a 24-2 cluster and its implication in human diseases. Mol Cancer 9: 232, 2010.

8. Saumet A, Vetter G, Bouttier M, et al: Transcriptional repression of microRNA genes by PML-RARA increases expression of key cancer proteins in acute promyelocytic leukemia. Blood 113: 412-421, 2009.

9. Xi Y, Shalgi R, Fodstad O, Pilpel Y and Ju J: Differentially regulated micro-RNAs and actively translated messenger RNA transcripts by tumor suppressor p53 in colon cancer. Clin Cancer Res 12: 2014-2024, 2006.

10. Volinia S, Calin GA, Liu CG, et al: A microRNA expression signature of human solid tumors defines cancer gene targets. Proc Natl Acad Sci USA 103: 2257-2261, 2006.

11. Dai Y, Sui W, Lan H, Yan Q, Huang H and Huang Y: Comprehensive analysis of microRNA expression patterns in renal biopsies of lupus nephritis patients. Rheumatol Int 29: 749-754, 2009.

12. Kozaki K, Imoto I, Mogi S, Omura K and Inazawa J: Exploration of tumor-suppressive microRNAs silenced by DNA hypermethylation in oral cancer. Cancer Res 68: 2094-2105, 2008.

13. Prueitt RL, Yi M, Hudson RS, et al: Expression of microRNAs and protein-coding genes associated with perineural invasion in prostate cancer. Prostate 68: 1152-1164, 2008.

14. Acunzo M, Romano G, Palmieri D, et al: Cross-talk between MET and EGFR in non-small cell lung cancer involves miR-27a and Sprouty2. Proc Natl Acad Sci USA 110: 8573-8578, 2013.

15. Wang W, Cheng B, Miao L, Mei Y and Wu M: Mutant p53-R273H gains new function in sustained activation of EGFR signaling via suppressing miR-27a expression. Cell Death Dis 4: e574, 2013. 
16. Zhang H, Li M, Han Y, et al: Down-regulation of miR-27a might reverse multidrug resistance of esophageal squamous cell carcinoma. Dig Dis Sci 55: 2545-2551, 2010.

17. Yu C, Chen $\mathrm{K}$, Zheng $\mathrm{H}$, et al: Overexpression of astrocyte elevated gene-1 (AEG-1) is associated with esophageal squamous cell carcinoma (ESCC) progression and pathogenesis. Carcinogenesis 30: 894-901, 2009.

18. Reyes M, Lund T, Lenvik T, Aguiar D, Koodie L and Verfaillie CM: Purification and ex vivo expansion of postnatal human marrow mesodermal progenitor cells. Blood 98: 2615-2625, 2001.

19. Edgar R, Domrachev M and Lash AE: Gene expression Omnibus: NCBI gene expression and hybridization array data repository. Nucleic Acids Res 30: 207-210, 2002.

20. Mathe EA, Nguyen GH, Bowman ED, et al: MicroRNA expression in squamous cell carcinoma and adenocarcinoma of the esophagus: associations with survival. Clin Cancer Res 15: 6192-6200, 2009.
21. Campbell PM, Groehler AL, Lee KM, Ouellette MM, Khazak V and Der CJ: K-Ras promotes growth transformation and invasion of immortalized human pancreatic cells by Raf and phosphatidylinositol 3-kinase signaling. Cancer Res 67: 2098-2106, 2007.

22. Downward J: Targeting RAS signalling pathways in cancer therapy. Nat Rev Cancer 3: 11-22, 2003.

23. Johnson SM, Grosshans $\mathrm{H}$, Shingara J, et al: RAS is regulated by the let-7 microRNA family. Cell 120: 635-647, 2005.

24. Yu S, Lu Z, Liu C, et al: miRNA-96 suppresses KRAS and functions as a tumor suppressor gene in pancreatic cancer. Cancer Res 70: 6015-6025, 2010.

25. Lyronis ID, Baritaki S, Bizakis I, Krambovitis E and Spandidos DA: K-ras mutation, HPV infection and smoking or alcohol abuse positively correlate with esophageal squamous carcinoma. Pathol Oncol Res 14: 267-273, 2008. 